\title{
Tracheostomy Practices for Mechanically Ventilated Patients in Malawi
}

\author{
Meghan Prin ${ }^{1} \cdot$ Alex Kaizer $^{2} \cdot$ Jesus Cardenas $^{3} \cdot$ Onias Mtalimanja $^{4} \cdot$ Clement Kadyaudzu $^{4} \cdot$ \\ Anthony Charles ${ }^{5}$. Adit Ginde ${ }^{6}$
}

Accepted: 7 May 2021 / Published online: 2 June 2021

(C) Société Internationale de Chirurgie 2021

\begin{abstract}
Background Tracheostomy is used for patients who require prolonged mechanical ventilation. Extensive research has described the provision and optimal timing of tracheostomy, but very little describes tracheostomy utilization in low- and middle-income countries, particularly in sub-Saharan Africa.

Methods This prospective cohort study describes patients admitted to the intensive care unit (ICU) of a tertiary hospital in Malawi who received tracheostomy versus those who did not, with a primary outcome of hospital mortality. We performed subgroup analysis of patients with severe head injuries.

Results The analysis included 451 patients admitted to the study ICU between September 2016 and July 2018. Overall hospital mortality was $40 \%$ for patients who received tracheostomy and $63 \%$ for patients who did not. Logistic regression modeling revealed an odds ratio (OR) of 0.34 (95\% CI 0.18-0.64) for hospital mortality among patients who received tracheostomy versus those who did not $(\mathrm{p}<0.001)$. Standardized mortality ratio weighting revealed an odds ratio of $0.81(95 \%$ CI $0.65-0.99, \mathrm{p}<0.001)$ for hospital death among patients who received tracheostomy versus those who did not. In the subgroup excluding severe head injury, both ICU (50\%) and hospital mortality (75\%) were higher overall, but hospital mortality was not more common for patients with tracheostomy versus without (OR $1.28,95 \%$ CI $0.94-1.74, \mathrm{p}=0.104)$.

Conclusions Tracheostomy is not associated with hospital mortality in a Malawi ICU cohort, but these results are affected by the presence of head injury. Research may focus on home tracheostomy care given the lack of hospital discharge options for patients in austere settings.
\end{abstract}

Supplementary Information The online version contains supplementary material available at https://doi.org/10.1007/s00268021-06176-3.

Meghan Prin

meghan.prin@cuanschutz.edu

Alex Kaizer

alex.kaizer@cuanschutz.edu

Jesus Cardenas

jesus.cardenas@cuanschutz.edu

Onias Mtalimanja

ofmtalimanja@yahoo.com

Clement Kadyaudzu

kadyaudzu@yahoo.com
Anthony Charles

anthony_charles@med.unc.edu

Adit Ginde

adit.ginde@cuanschutz.edu

1 Department of Anesthesiology, University of Colorado Anschutz Medical Center, 12401 E. 17th Avenue, 7th Floor, Aurora, CO 80045, USA

2 Department of Biostatistics and Informatics, Colorado School of Public Health, Aurora, CO, USA 


\section{Introduction}

Tracheostomy is indicated for critically ill patients in need of prolonged mechanical ventilatory support. It reduces the risks associated with oral or nasal endotracheal intubation, including a decreased risk of ventilator-associated pneumonia $[1,2]$, earlier transfer patients out of the intensive care unit (ICU) [3], and improved patient comfort and mobility due in part to the ability to use the vocal cords and epiglottis (e.g., speaking and swallowing) [4]. While there has been extensive research on the optimal timing of tracheostomy for different ICU patient populations in highincome settings [3], few studies to date have described tracheostomy practice patterns for adult ICU patients in low- and middle-income countries (LMIC) such as those of sub-Saharan Africa. In an effort to target this literature gap, this study describes tracheostomy practices and outcomes for ICU patients at a referral hospital in Malawi.

\section{Methods}

This was a secondary analysis of a prospective dataset created to evaluate critical care illness severity at Kamuzu Central Hospital $(\mathrm{KCH})$ in Lilongwe, Malawi, from September 2016 to July 2018. The study protocol was registered at researchregistry.com under protocol 4330 and has been detailed in other publications [5]. It was developed a priori and approved by the National Health Sciences Research Council of Malawi and the Institutional Review Board of the associated American academic medical center, and the requirement for written informed consent was waived. The timing of the study recruitment was based on funding limitations; no power calculations were undertaken. The results are reported in line with the Strengthening the Reporting of Cohort Studies in Surgery (STROCSS) criteria [6].

Malawi is a country in southeastern Africa with a population of about 18 million people, a life expectancy of 63.8 years, and a Human Development Index rank of 170 out of 187 countries [7]. It is the fourth poorest country in sub-Saharan Africa [8]. KCH is a referral hospital in the central region of Malawi with a catchment area of

3 University of Colorado School of Medicine, Aurora, CO, USA

4 Department of Anesthesiology, Kamuzu Central Hospital, Lilongwe, Malawi

5 Department of Surgery, University of North Carolina At Chapel Hill, Chapel Hill, NC, USA

6 Department of Emergency Medicine, University of Colorado Anschutz Medical Center, Aurora, CO, USA approximately 6 million. The ICU at $\mathrm{KCH}$ is a 5-bed unit which offers a 1:1 nurse-to-patient ratio during the day and a 1:2 or higher nurse-to-patient ratio at night. Resources include continuous non-invasive vital sign monitoring, mechanical ventilation, and intravenous medication infusions. Clinical care in the ICU is directed by clinical officers trained in anesthesiology; these officers make initial recommendations regarding the need for tracheostomy. Tracheostomies are performed in this unit by consultants in otolaryngology or general surgery.

These study data were collected prospectively by research staff trained for ICU data collection. Variables collected included vital signs and laboratory measurements at the time of ICU admission, treatments utilized in ICU, and the dates of ICU or hospital discharge. All patients admitted to the ICU who required mechanical ventilation were eligible for inclusion. Exclusion criteria included tracheostomy placement before ICU admission, readmission to ICU during the same hospitalization, and missing primary outcome or multiple key data points (Fig. 1). Missing data accounted for $<3 \%$ of data for all variables included in this analysis.

The primary outcome was in-hospital mortality. We first described the cohort, stratified by the provision of tracheostomy. We performed the chi-square test, students' t-test, and analysis of variance to compare groups, as appropriate. We used logistic regression to evaluate the association between tracheostomy and in-hospital mortality, adjusting for the covariates of the MIME score chosen a priori based on their association with hospital mortality in this population [5]. Given imbalances between the groups, propensity scores were obtained to account for potential confounding. A propensity score was calculated to illustrate the probability of a patient being in the tracheostomy group given age, admitting service, systolic blood pressure and altered mental status at ICU admission, and fever during the ICU course. Using the propensity score, we then derived the standardized mortality ratio weight (SMRW) for each patient to reduce the imbalances between the groups. After accounting for SMRW, group differences were evaluated by the SMRW proportions and the standardized differences. All covariates had standardized differences $<0.10$, and regression analysis was restricted to those on common support.

Given our previous research which showed exceptionally high mortality among patients admitted to the ICU with a severe brain injury [9], we then repeated our analyses excluding these patients. Continuous variables are presented as means with standard deviations (SD); categorical variables are presented as number and proportion of the total. 95\% confidence intervals (CIs) are provided as needed. All statistical analyses were 
performed with Stata SE 14.2 (StataCorps, College Station, TX).

\section{Results}

During the study period, 498 patients were admitted to the study ICU, and 451 were included in the final cohort. (Fig. 1) The clinical characteristics of patients who underwent tracheostomy versus those who did not are summarized in Table 1. Overall, 55 patients received a tracheostomy and all were elective. Tracheostomies were performed using an open surgical approach at the bedside, under intravenous anesthesia. No early complications in tracheostomy placement or use were described. This study did not include assessment for tracheostomy complications later in the hospital course outside the ICU setting.

The majority of the cohort $(69 \%)$ was male, and most patients had been admitted to ICU for severe head injuries or meningitis $(n=40)$. Patients who underwent tracheostomy were mechanically ventilated longer than patients who did not (mean 14 days (SD 8) versus mean 3 days (SD 4), p < 0.001). Patients who underwent tracheostomy were mechanically ventilated for a mean of 9 days (SD 5, median 10) before receiving the tracheostomy. Hospital mortality was $40 \%$ for patients who received tracheostomy in ICU, and $63 \%$ for patients who

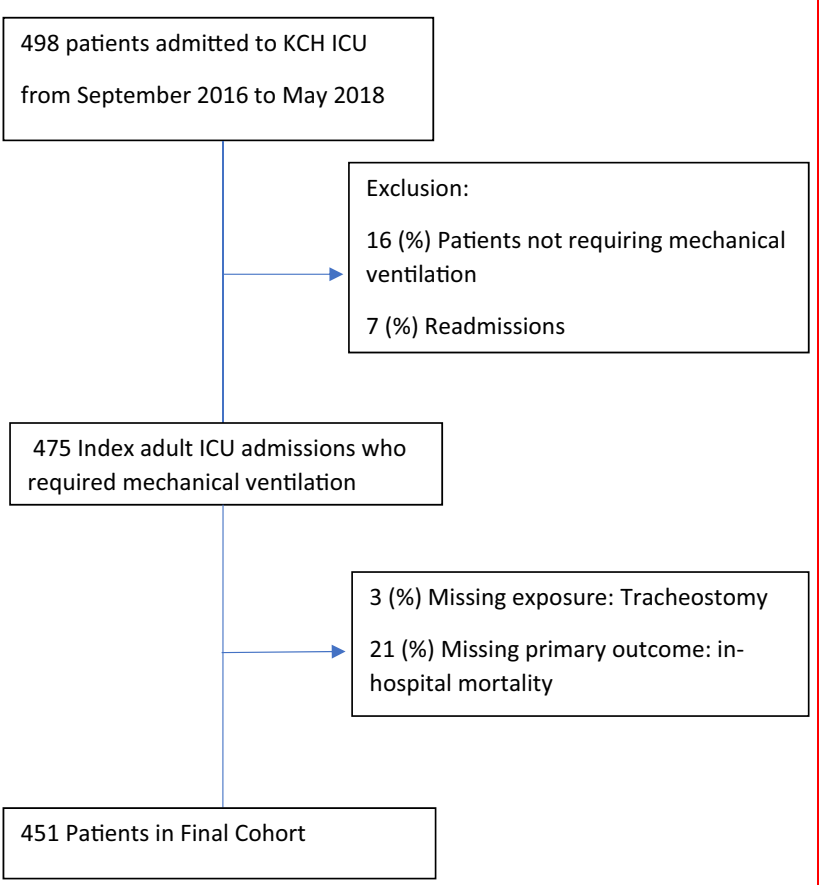

Fig. 1 Flowchart of patient selection did not. Logistic regression modeling with adjustment for the components of the MIME model revealed an odds ratio (OR) of 0.34 (95\% CI 0.18-0.64) for hospital mortality among patients who received tracheostomy versus those who did not $(\mathrm{p}<0.001)$. In the standardized mortality ratio weighted cohort, the odds ratio for hospital death among patients who received tracheostomy versus those who did not was 0.81 (95\% CI 0.65-0.99, p < 0.001).

We repeated our analyses in a subset of the study cohort which excluded patients with severe brain injuries $(n=97)$. In this analysis, a total of 354 patients were included and 16 underwent tracheostomy. About half of patients who received a tracheostomy were male (44\%), and ICU admission diagnoses for these patients included acute respiratory failure $(n=7)$, "high spinal" or neuraxial anesthetic complications, Guillan-Barre, postoperative monitoring, and shock. Both ICU mortality (50\%) and hospital mortality (75\%) were higher in this group of tracheostomy patients compared to the larger group which included head injuries. (Supplement Table 1) In the standardized mortality ratio weighted analysis, the odds of hospital death was not higher for patients with tracheostomy versus those without (OR $1.28 \quad(95 \%$ CI $0.94-1.74, \mathrm{p}=0.104)$.

\section{Discussion}

This descriptive study of tracheostomy practices at a tertiary hospital in Malawi indicates that tracheostomy was provided to $12 \%$ of patients who required ICU admission, the majority of which had a severe head injury. Tracheostomy was provided to patients after a mean 9 days (SD 5) of mechanical ventilation, which falls in line with international clinical practice guidelines [10]. While tracheostomy initially appeared to be associated with hospital mortality, our analysis excluding severe head injured patients demonstrates that severe head injury likely confounds the association of tracheostomy with hospital mortality in this setting.

Endotracheal intubation is a widely used intervention to provide mechanical ventilation to patients with pulmonary illnesses and/or an inability to protect the airway. Prolonged endotracheal intubation, however, is associated with longer durations of mechanical ventilation (and associated risks of pneumonia) and an inability to eat or speak normally [1-3]. A tracheostomy provides long-term access to a protected airway. The short tracheostomy tube has significantly lower resistance than a standard endotracheal tube, is more comfortable by avoidance of gag or cough reflexes, and allows the patient to swallow and speak. Tracheostomy is not without risks, and complications can be classified as early (e.g., bleeding) or late (e.g., 
Table 1 Clinical characteristics and hospital course for ICU patients who required mechanical ventilation at a referral hospital in Malawi, stratified by the provision of tracheostomy

\begin{tabular}{|c|c|c|c|}
\hline Variable & Received tracheostomy & Did not receive tracheostomy & $p$ value \\
\hline Total ICU admissions, $n(\%)$ & $55(12)$ & $396(88)$ & - \\
\hline Age (years), mean (SD) & $29(13)$ & $30(17)$ & 0.745 \\
\hline \multicolumn{4}{|l|}{ Sex, $n(\%)$} \\
\hline Female & $17(31)$ & $220(56)$ & \multirow[t]{2}{*}{0.001} \\
\hline Male & $37(69)$ & $176(44)$ & \\
\hline \multicolumn{3}{|l|}{ Hospital location before ICU admission, $n(\%)$} & \multirow[t]{5}{*}{$<0.001$} \\
\hline Emergency room ${ }^{\mathrm{a}}$ & $27(49)$ & $63(16)$ & \\
\hline HDU & $14(25)$ & $93(23)$ & \\
\hline Operating room & $11(20)$ & $182(46)$ & \\
\hline Ward & $3(5)$ & $41(10)$ & \\
\hline \multicolumn{4}{|l|}{ ICU Admitting Service, $n(\%)$} \\
\hline General surgery & $20(36)$ & $176(44)$ & \multirow[t]{3}{*}{$<0.001$} \\
\hline OBGyn & $5(9)$ & $89(22)$ & \\
\hline Medicine/pediatrics & $30(55)$ & $117(30)$ & \\
\hline Recent surgery in hospital course, $n(\%)$ & $17(31)$ & $241(61)$ & $<0.001$ \\
\hline Total MEWS at ICU admission, mean (SD) & $6(3)$ & $7(5)$ & 0.0312 \\
\hline Length of mechanical ventilation (days), mean (SD) & $14(8)$ & $3(4)$ & $<0.001$ \\
\hline Length of mechanical ventilation before tracheostomy (days), mean (SD) & $9(5)$ & NA & NA \\
\hline Length of stay in ICU (days), mean (SD) & $16(9)$ & $3(4)$ & $<0.001$ \\
\hline ICU mortality, $n(\%)$ & $14(25)$ & $234(59)$ & $<0.001$ \\
\hline Hospital mortality, $n(\%)$ & $22(40)$ & $250(63)$ & 0.001 \\
\hline
\end{tabular}

ICU Intensive care unit, $S D$ standard deviation, $H D U$ high-dependency unit, $O B G y n$ obstetrics and gynecology, MEWS Modified Early Warning Score

${ }^{a}$ Emergency room includes transfers from outside hospitals

tube obstructions, dislodgments, and nosocomial lung infections). Nevertheless, tracheostomy is considered safe and is relatively well tolerated even among complex patient populations.

Although tracheostomy is essential surgery within a healthcare system, literature describing its use in the lowincome countries of sub-Saharan Africa is scarce. One report by Charles et al. prospectively characterized all Otolaryngology referrals at a tertiary hospital in Rwanda over an 8-month period and found that the majority of patients who required tracheostomy were critically ill and $75 \%$ had a severe head injury [11]. A retrospective study conducted in northern Nigeria found that $26 \%$ of tracheostomies were provided to patients with traumatic injuries [12]. Our findings in Malawi are consistent with these reports, although our data are more granular. What differentiates our cohort from those in Nigeria and Rwanda is that all tracheostomies in this study were elective. This may suggest that our study site has the capacity to provide tracheostomies as needed (avoiding the need for emergency airway management), or alternatively that emergency tracheostomies occur outside the ICU setting at our study site.
There are numerous studies describing the optimal timing of tracheostomy. The TracMan study was one of the largest of these studies and found that tracheostomy within four days of ICU admission was not associated with improved 30-day mortality or other outcomes (e.g., ICU mortality, duration of mechanical ventilation) when compared to later tracheostomy [13]. A subsequent metaanalysis found that tracheostomy within seven days of intubation is recommended for patients with prolonged intubation [14]. In this study population, the mean length of mechanical ventilation before tracheostomy was 9 days, with a standard deviation of 5 days. Despite severe resource limitations, these data demonstrate that the provision of tracheostomy in this setting is within international guidelines. Although beyond the scope of this paper, further research should explore whether outcomes vary based on the timing of tracheostomy in this patient population and setting.

It is not surprising that severe head injury confounded the association of tracheostomy with hospital mortality, as severe head injury is a known predictor of the need for tracheostomy and is associated with especially high mortality at this study site [9]. In Malawi, it is common 
practice to provide tracheostomy to patients who may require prolonged intubation so that they can more easily be transitioned to a high-dependency unit (HDU) or the ward. This practice allows for the re-opening of ICU beds in a setting where ICU availability is only 1 per million population, but also means transitioning severely injured patients to a lower level of care. More research is needed to determine the best practices for patients who require prolonged intubation in the setting of extremely scarce ICU resources.

Another major difference in the continuum of care for survivors of prolonged mechanical ventilation in Malawi is hospital discharge destination. Survivors of critical illness in high-income countries are often ultimately discharged from the hospital to long-term acute care facilities or skilled nursing facilities. Healthcare systems in LMICs generally do not offer these discharge destinations. Because of this, almost all pulmonary rehabilitation must occur either in the hospital or in the home. Investments in both arenas may be especially beneficial for patients in LMICs. A multidisciplinary team of otolaryngologists, respiratory therapists, tracheostomy nurses, medical illustrators, and global health educators in Rwanda created an open access training course for tracheostomy care which improved nurses' and resident doctors' confidence in tracheostomy care [15]. This type of course could be adapted for use by family members to improve its applicability in the home in austere settings.

This study has several limitations. First, this study was conducted within a single center which limits its generalizability. Data describing long-term outcomes, including late tracheostomy complications beyond the ICU stay, were not available. Nevertheless, these prospective data are more granular than other reports from LMICs and may serve as a valuable launching point for rigorous discussions of tracheostomies in resource-limited settings.

\section{Conclusions}

Tracheostomy is essential surgery, and in our study ICU in Malawi, it was provided to $12 \%$ of patients over two years. Hospital mortality did not differ between patients who received tracheostomy versus those who did not, although this was confounded by the presence of a severe head injury. Future research may focus on the timing of tracheostomy, but in resource-limited settings a focus on tracheostomy care in the hospital and at home may be more appropriate.
Funding This study was supported in part by the NIH Fogarty International Center Postdoctoral Research Fellowship to Dr. Prin. The contents of the manuscript are solely the responsibility of the authors and do not necessarily reflect the official views of the funding agencies.

\section{References}

1. Siempos II, Ntaidou TK, Filippidis FT et al (2015) Effect of early versus late or no tracheostomy on mortality and pneumonia of critically ill patients receiving mechanical ventilation: a systematic review and meta-analysis. Lancet Respir Med 3:150-158

2. Nseir S, Di Pompeo C, Jozefowicz E et al (2007) Relationship between tracheotomy and ventilator-associated pneumonia: a case control study. Eur Respir J 30:314-320

3. Andriolo BN, Andriolo RB, Saconato $\mathrm{H}$ et al (2015) Early versus late tracheostomy for critically ill patients. Cochrane Database Syst Rev 1(1):CD007271

4. Whitmore KA, Townsend SC, Laupland KB (2020) Management of tracheostomies in the intensive care unit: a scoping review. BMJ Open Respir Res 7:e000651

5. Prin M, Pan S, Kadyaudzu C et al (2018) Development of a Malawi Intensive care Mortality risk Evaluation (MIME) model, a prospective cohort study. Int J Surg 60:60-66

6. Agha R, Borrelli M, Vella-Baldacchino M et al (2017) The STROCSS statement: Strengthening the Reporting of Cohort Studies in Surgery. Int J Surg 46:198-202

7. UN (2016) Human Development Reports. United Nations Development Programme, accessed at http://hdr.undp.org/sites/ default/files/2016_human_development_report.pdf. Accessed 10 Jan 2021

8. Gregson J, The Richest Countries in the World (2018) In: International Monetary Fund, World Economic Outlook Database, accessed at https://www.gfmag.com/global-data/economicdata/richest-countries-in-the-world. Accessed 4 Dec 2020

9. Prin M, Quinsey C, Kadyaudzu C et al (2019) Brain death in lowincome countries: a report from Malawi. Trop Doct 49:107-112

10. Trouillet JL, Collange O, Belafia F et al (2018) Tracheotomy in the intensive care unit: Guidelines from a French expert panel: The French Intensive Care Society and the French Society of Anaesthesia and Intensive Care Medicine. Anaesth Crit Care Pain Med 37:281-294

11. Charles N, Mukara KB (2018) Indications and immediate outcomes of tracheostomy in Rwanda. Annals of African Surgery $15(2)$

12. Alabi BS, Afolabi OA, Dunmade AD et al (2018) Indications and outcome of tracheostomy in Ilorin, North Central Nigeria: 10 years review. Ann Afr Med 17:1-6

13. Young D, Harrison DA, Cuthbertson BH et al (2013) Effect of early vs late tracheostomy placement on survival in patients receiving mechanical ventilation: the TracMan randomized trial. JAMA 309:2121-2129

14. Adly A, Youssef TA, El-Begermy MM et al (2018) Timing of tracheostomy in patients with prolonged endotracheal intubation: a systematic review. Eur Arch Otorhinolaryngol 275:679-690

15. Sandler ML, Ayele N, Ncogoza I et al (2020) Improving Tracheostomy Care in Resource-Limited Settings. Ann Otol Rhinol Laryngol 129:181-190

Publisher's Note Springer Nature remains neutral with regard to jurisdictional claims in published maps and institutional affiliations. 